\title{
LOCALIZED VIBRATIONAL MODES OF INTERSTITIAL OXYGEN IN $\mathrm{Si}_{x} \mathrm{Ge}_{1-x}$ ALLOYS
}

\author{
M. LORENC* AND J. HUMLÍ́ČEK \\ Laboratory of Thin Films and Nanostructures \\ Department of Solid State Physics, Faculty of Science, Masaryk University \\ Kotlářská 2, 61137 Brno, Czech Republic
}

\begin{abstract}
We study infrared-active vibrations of interstitial oxygen in Czochralski-grown samples of $\mathrm{Si}_{x} \mathrm{Ge}_{1-x}$ alloys with the silicon content from 0 to 1 between room and liquid nitrogen temperature. The measurements were performed on a large series of samples including single crystals of Si and Si-rich alloys, and polycrystalline samples of alloys with a higher Ge content. We focus on quantitative data on the compositional and temperature evolution of the strongest absorption band above $1100 \mathrm{~cm}^{-1}$, related to the asymmetric stretching of the $\mathrm{Si}_{2} \mathrm{O}$ pseudomolecule. We have also observed a pronounced Fano-like resonance centered at $520 \mathrm{~cm}^{-1}$.
\end{abstract}

PACS numbers: 78.30.Am, 63.20.Pw

In Czochralski-grown silicon, germanium, and $\mathrm{Si}_{x} \mathrm{Ge}_{1-x}$ alloy crystals, oxygen exists primarily as an interstitial defect $\left(\mathrm{O}_{\mathrm{i}}\right)$. The infrared-active vibrations of $\mathrm{O}_{\mathrm{i}}$ in $\mathrm{Si}$ and $\mathrm{Ge}$ have been studied many times. Recently, the structure, dynamics and infrared polarizability have been revised for both $\mathrm{Si}: \mathrm{O}$ and $\mathrm{Ge}: \mathrm{O}$, see Refs. [1-3] and references therein.

In this paper, we report the basic characteristics of the most prominent $\mathrm{O}_{i}$ absorption bands in the alloys. The results are obtained from a comprehensive set of measurements on 19 samples, covering the whole compositional range from pure $\mathrm{Si}$ to pure $\mathrm{Ge}$, performed in the temperature range of $85-300 \mathrm{~K}$. Our main aim is to provide quantitative data on the compositional and temperature evolution of the strongest, localized-vibration band related to the asymmetric stretching of the $\mathrm{Si}_{2} \mathrm{O}$ pseudomolecule. This band is located at $c a .1106 \mathrm{~cm}^{-1}$ in Si at room temperature, and is known to exhibit a composite structure at low temperatures [4-6]. We also report on the structure related to the lower-frequency infrared-active mode, occurring at about $520 \mathrm{~cm}^{-1}$ in pure Si.

The measurements have been performed on single-crystal and polycrystalline samples grown by Czochralski (CZ) method. The samples were not intentionally doped; the free-hole concentration at room temperature was typically $c a .10^{15} \mathrm{~cm}^{-3}$. The oxygen concentration was about $10^{18} \mathrm{~cm}^{-3}$ throughout the

*e-mail: mlo@physics.muni.cz 
whole compositional range. The sample thickness ranged from 1 to $2 \mathrm{~mm}$. Transmission measurements have been performed with a FTIR spectrometer Bruker IFS55, with the spectral resolution of $1 \mathrm{~cm}^{-1}$. The background of two- and three-phonon absorption of the defect-free silicon lattice has been measured on a float-zone (FZ) sample, having a negligible concentration of $\mathrm{O}_{\mathrm{i}}$. Another source of the absorption background is the free-hole plasma, which can be easily identified due to its monotonic Drude-like increase towards lower frequencies.

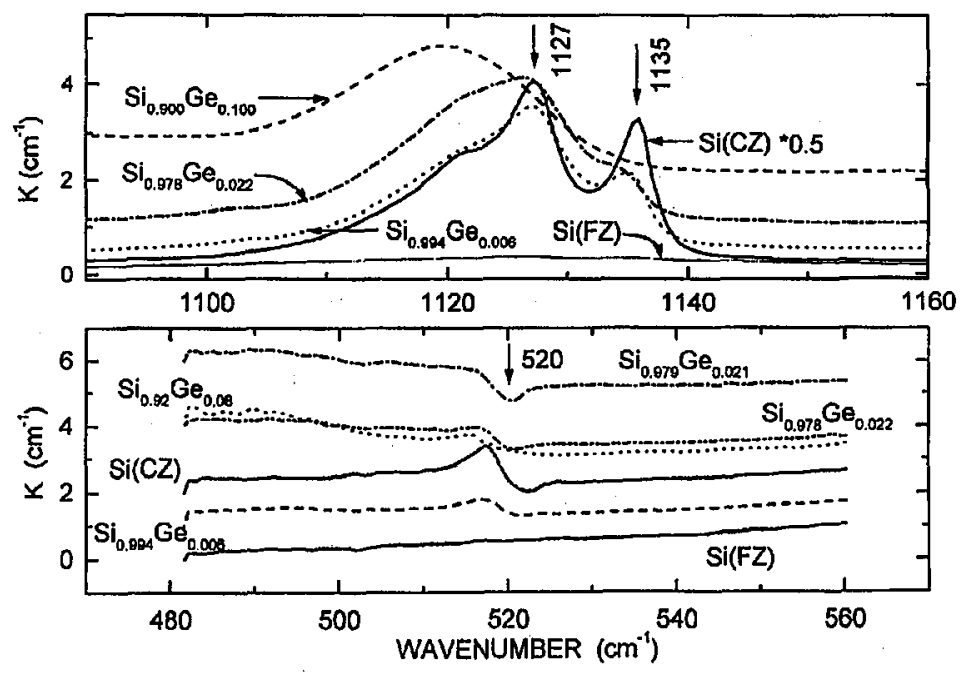

Fig. 1. Low-temperature $(85 \mathrm{~K})$ spectra of several silicon-rich samples in the range of symmetric (lower panel) and asymmetric (upper panel) stretching vibrations of interstitial oxygen.

In Fig. 1 there is an overview of the spectra of absorption coefficient for silicon-rich alloys in two separate frequency ranges at $85 \mathrm{~K}$. The band above $1100 \mathrm{~cm}^{-1}$ shifts towards lower frequencies with increasing Ge content, becomes broader, and its fine structure is almost completely lost for $x \geq 10$ atomic \% Ge. It is related to the asymmetric stretching of the $\mathrm{Si}_{2}$ unit; the resonance frequency is expected to decrease with the substitution of at least one of the six adjacent silicon atoms with the heavier atom of Ge. We were able to identify this band for the Ge content up to $c a .80$ at.\%. The peak positions extracted from the data taken at several temperatures are listed in Table. Note the monotonic changes of the peak positions and their temperature coefficients, $a_{1}$, with changing the alloy composition.

The multiple-component spectra of the high-frequency band are shown in Fig. 2 for silicon and the 2.2 at.\% Ge alloy. In order to quantify the influence of alloying, we have fitted the spectra with a sum of Gauss-Lorentz (G-L) oscillators. The Gaussian and Lorentzians profiles of variable widths were convoluted and their parameters adjusted to the measured data. Besides the increase in the 


\section{TABLE}

Observed compositional dependence of the asymmetric stretching vibration of interstitial oxygen in $\mathrm{Si}_{x} \mathrm{Ge}_{1-x}$ alloys. Parameters of the best-fit linear regresion, $\nu(T)=a_{0}+a_{1}(300[\mathrm{~K}]-T)$, in the $85-300 \mathrm{~K}$ range.

\begin{tabular}{c|c|c|c|c}
\hline \hline $\boldsymbol{x}$ & $\begin{array}{c}\nu(300 \mathrm{~K}) \\
{\left[\mathrm{cm}^{-1}\right]}\end{array}$ & $\begin{array}{c}\nu(85 \mathrm{~K}) \\
{\left[\mathrm{cm}^{-1}\right]}\end{array}$ & $\begin{array}{c}a_{0} \\
{\left[\mathrm{~cm}^{-1}\right]}\end{array}$ & $\begin{array}{c}a_{1} \\
{\left[10^{-3} \mathrm{~cm}^{-1} \mathrm{~K}^{-1}\right]}\end{array}$ \\
\hline $1(\mathrm{Si})$ & 1106.5 & 1127.3 & $1106.4 \pm 1.0$ & $100 \pm 7$ \\
0.994 & 1107.8 & 1127.0 & $1107.7 \pm 1.1$ & $90 \pm 7$ \\
0.979 & 1107.5 & 1126.4 & $1106.3 \pm 1.3$ & $91 \pm 9$ \\
0.978 & 1106.3 & 1127.3 & $1107.0 \pm 0.9$ & $93 \pm 6$ \\
0.920 & 1104.4 & 1120.7 & $1104.4 \pm 0.3$ & $74 \pm 2$ \\
0.900 & 1102.6 & 1119.6 & $1102.5 \pm 0.3$ & $80 \pm 1$ \\
0.890 & 1105.0 & 1120.7 & $1104.8 \pm 0.4$ & $74 \pm 3$ \\
0.840 & 1102.6 & 1118.9 & $1102.5 \pm 0.6$ & $75 \pm 4$ \\
0.790 & 1101.8 & 1116.5 & $1101.9 \pm 0.4$ & $71 \pm 3$ \\
0.770 & 1100.7 & 1117.0 & $1100.2 \pm 0.8$ & $75 \pm 6$ \\
0.710 & 1099.3 & 1116.1 & $1099.2 \pm 0.3$ & $78 \pm 2$ \\
0.580 & 1095.9 & 1112.6 & $1095.8 \pm 0.4$ & $79 \pm 3$ \\
0.510 & 1091.8 & 1111.4 & $1091.8 \pm 0.2$ & $96 \pm 1$ \\
0.390 & 1091.6 & 1111.3 & $1092.0 \pm 1.2$ & $89 \pm 8$ \\
0.208 & 1090.8 & 1109.8 & $1090.8 \pm 1.0$ & $86 \pm 5$
\end{tabular}

Gaussian widths with increasing Ge content, the data show an interesting behavior of the band positions. The three main components of $\mathrm{Si}$ are located at $1123.0 \pm 0.1,1127.3 \pm 0.1$ and at $1135.6 \pm 0.1 \mathrm{~cm}^{-1}$. Upon adding 2.2 at. $\% \mathrm{Ge}$, the band positions become $1120.6 \pm 0.2,1127.3 \pm 0.3$ and $1134.5 \pm 0.2$. In addition, we find a new weak band at $1101.5 \pm 1.0 \mathrm{~cm}^{-1}$. While the two outer components of the strong triplet shift towards lower frequencies, the $1127 \mathrm{~cm}^{-1}$ band stays constant. This behavior has been confirmed by separating the individual bands by numerical differentiation of the measured spectra in a broader range of compositions. Assuming the rotational origin of the composite structure $[4,7]$, we are able to estimate the changes in the bonding due to the presence of $\mathrm{Ge}$ in $\mathrm{Si}$ matrix. Namely, the difference between a fundamental vibration at about $1135 \mathrm{~cm}^{-1}$ and the nearest lower band is $8.3 \mathrm{~cm}^{-1}$ in pure $\mathrm{Si}$ and $7.2 \mathrm{~cm}^{-1}$ in $\mathrm{Si}_{0.978} \mathrm{Ge}_{0.022}$. This corresponds to a possible decrease in $\mathrm{Si}-\odot-\mathrm{Si}$ bond angle of about $10 \%$ due to the substitution of one $\mathrm{Si}$ atom by the heavier and larger Ge atom.

The low-frequency range of Fig. 1 is dominated by a strongly asymmetric spectral structure centered at $520 \mathrm{~cm}^{-1}$. In some of the samples, the structure is actually an antiresonance, see the 2.1 at.\% data of Fig. 1 . We have noticed a significant correlation of this line shape with the strength of the background ab- 

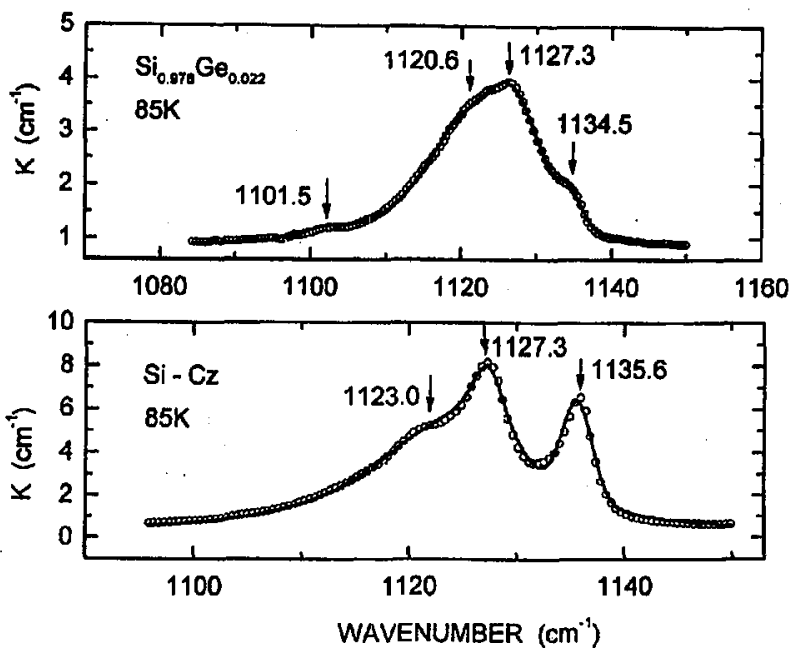

Fig. 2. The best fit line shapes (solid lines) of the measured spectra at $85 \mathrm{~K}$ (open symbols) for silicon (lower panel) and the alloy of 2.2 at.\% germanium (upper panel). The arrows indicate the positions of the individual G-L bands.

sorption. Since a part of the background absorption is due to the free-hole plasma, we assign tentatively the observed spectra to a Fano-type resonance between the dipole-forbidden, one-phonon absorption in the silicon lattice and the continuum of intravalence electronic transitions. The latter spectra have been identified reliably at higher $p$-type dopings, see, e.g., Refs. [8, 9] and references therein. This assignment is further supported by the weak dependence of the resonance position on the Ge content. In fact, the oxygen-related threshold below $520 \mathrm{~cm}^{-1}$ in Si has been interpreted as the vibration of the $\mathrm{Si}$ lattice induced by the bond-breaking $\mathrm{O}_{\mathrm{i}}[2]$. This spectral structure shifts rather strongly to lower frequencies $\left(\approx 330 \mathrm{~cm}^{-1}\right)$ in $\mathrm{Ge}: \mathrm{O}[1]$.

In conclusion, we have identified the strongest effects of alloying on the infrared spectra of interstitial oxygen. Further work is conducted to extend these results.

The work has been supported by the grant VS96102 of the Ministry of Education of Czech Republic.

\section{References}

[1] E. Artacho, F. Ynduráin, B. Pajot, R. Ramírez, C.P. Herrero, L.I. Khirunenko, K.M. Itoh, E.E. Haller, Phys. Rev. B 56, 3820 (1997).

[2] E. Artacho, A. Lizón, F. Ynduráin, Phys. Rev. B 51, 7862 (1995).

[3] H. Yamada-Kaneta, C. Kaneta, T. Ogava, Phys. Rev. B 42, 9650 (1990).

[4] B. Pajot, J. Phys. Chem. Solids 28, 77 (1967).

[5] B. Pajot, H.J. Stein, B. Cales, C. Naud, J. Electrochem. Soc. 132, 3034 (1985).

[6] R. Jones, A. Umerski, S. Öberg, Phys. Rev. B 45, 11321 (1992). 
[7] N. Aichele, U. Gommel, K. Lassmann, F. Maier, F. Zeller, E.E. Haller, K.M. Itoh, L.I. Khirunenko, V. Shakhovtsov, B. Pajot, E. Fogarassy, H. Muessig, Mater. Sci. Forum 258-263, 47 (1997).

[8] V.I. Belitsky, M. Cardona, Solid State Commun. 100, 837 (1996).

[9] J. Humlíček, Thin Solid Films 313-314, 656 (1998). 